\title{
Abrechnungstipp
}

\section{Neuere GOÄ-Leistungen „analog“ abrechnen}

\author{
In der zurzeit gültigen GOÄ, die 1982 zusammengestellt wurde, sind viele neue \\ medizinische, diagnostische oder therapeutische Leistungen nicht gelistet. Sie haben \\ aber dennoch die Möglichkeit diese Leistung abzurechnen.
}

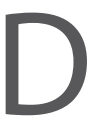

ie Leistungen, die in der aktuell gültigen GOÄ fehlen, sind mittlerweile zahlreich, aber natürlich trotzdem abrechenbar. Im Text des $\$ 6$ Abs. 2 der GOÄ heißt es: „Selbstständige ärztliche Leistungen, die in das Gebührenverzeichnis nicht aufgenommen sind, können entsprechend einer nach Art, Kosten- und Zeitaufwand gleichwertigen Leistung des Gebührenverzeichnisses berechnet werden." Sie nehmen also eine ähnliche oder vergleichbare Leistung und rechnen ihre "neue“ Leistung „analog“ der anderen Leistung ab. Zur Unterscheidung werden diese "Analogziffern“ mit einem großen, vorangestellten „A“ ausgewiesen. Ein hierbei hilfreiches Analogziffernverzeichnis hält die Bundesärztekammer auf ihrem Portal bereit: http://www.bundesaerztekammer.de/downloads/GOAE_ Mai_2012_Auszug_Analogverzeichnis_ Deutscher_Aerzte-Verlag.pdf. Diese „offiziellen“ Analogziffern stellen auch bei Versicherern kein Problem dar und werden in der Regel ohne Diskussion vergütet.

\section{Analogziffern selbst erstellen}

Wenn in der GOÄ nicht enthaltene Leistungen angewandt werden, sollte zunächst das Analogverzeichnis der GÖ̈ durchgesehen werden. Hier findet sich allerdings nur eine klassisch psychiatrische Leistung, nämlich die Nr. A 888: „Psychiatrische Behandlung zu Reintegration eines Erwachsenen mit psychopathologisch definiertem Krankheitsbild als Gruppenbehandlung (in Gruppen von 3 bis 8 Teilnehmern) durch syndrombezogene verbale Intervention als therapeutische Konsequenz aus den dokumentierten Ergebnissen der selbsterbrachten Leistung nach Nr. 801, Dauer mindestens 50 Minuten, je Teilnehmer und Sitzung, analog Nr. 887, 200 Punkte. “Analogziffern können jedoch auch selbst erstellt werden, nach folgenden Kriterien: Zunächst muss es eine der Art nach vergleichbare Leistung sein; man kann also keine therapeutischen analog diagnostischen Leistungen abrechnen. Auch ist eine operative Leistung nicht mit einer konservativen therapeutischen Leistung vergleichbar. Alle Vorgaben über eventuelle Mindestzeitangaben, Ausschlüsse, Bewertungen, maximale Abrechnungshäufigkeit pro Zeiteinheit (Behandlungsfall, Jahr) oder sonstige Einschränkungen der zu Hilfe genommenen Abrechnungsziffer gelten auch für die Analogziffer. Abweichungen sind nicht erlaubt. Beispielhaft seien genannt:

-Gebührenrahmen: Der Höchst- und Schwellenwert der ursprünglichen Leistung sind beizubehalten. Für eine ärztliche Leistung muss auch die Analogziffer eine ärztliche Leistung (mit den entsprechenden Multiplikatoren) sein. Der in den Abschnitten A, E und O GOÄ reduzierte Gebührenrahmen muss auch bei der neuen Ziffer greifen. _Ausschlüsse: Alle genannten Ausschlüsse der ursprünglichen Ziffer werden auf die Analogziffer übertragen.

— Abrechnungshäufigkeit: pro Sitzung, Behandlungsfall oder Krankheitsfall.

— Mindestzeiten müssen sich bei der ursprünglichen und der Analogziffer gleichen, im obigen Beispiel 50 Minuten.

_Kombinierbarkeit: Auch hier darf es keine Abweichungen geben. Eine auf die Nr. 3 sich beziehende Analogziffer besitzt dann die gleichen Einschränkungen wie die ausführliche Beratung.
_Anzahl der Gruppenteilnehmer: Auch sie muss identisch mit der originären Ziffer sein.

Im Kapitel M Laboratoriumsuntersuchungen sind die Möglichkeit der Analogabrechnung und entsprechende Modalitäten im Punkt 8 noch einmal ausdrücklich erwähnt: „Für die analoge Abrechnung einer nicht aufgeführten selbstständigen Laboruntersuchung ist die nach Art, Kosten- und Zeitaufwand zutreffendste Gebührennummer aus den Abschnitten M II bis $M I V z u$ verwenden. In der Rechnung ist diese Gebührennummer durch Voranstellen des Buchstabens „A“ als Analogabrechnung zu kennzeichnen."

Und im $\$ 12$ Abs. 4 der GOÄ ist noch einmal die erforderliche Kennzeichnung dieser Leistungen in der Rechnung hervorgehoben:,Wird eine Leistung nach $\$ 6$ Abs. 2 berechnet, ist die entsprechend bewertete Leistung für den Zahlungspflichtigen verständlich $z u$ beschreiben und mit dem Hinweis „entsprechend“ sowie der Nummer und der Bezeichnung der als gleichwertig erachteten Leistung zu versehen."

\section{Mit Fantasieziffern kommt man nicht weit}

Der Tatsache, dass die Bewertung mancher Leistung in der GOÄ heute den realen Bedingungen nicht mehr entspricht, ist nicht $\mathrm{zu}$ widersprechen. Sie erlaubt allerdings nicht, für in der GOÄ abgebildete Ziffern eigene Ziffern - auch keine Analogziffern - zu formulieren, nur weil die Honorarhöhe nicht den Vorstellungen entspricht. Eine Möglichkeit aus dieser Zwickmühle wäre beispielsweise die Anwendung eines höheren Multiplikators.

Dr. med. Heiner Pasch 